\title{
PROJECT INDICATORS VISUALIZATION USING AN INTERACTIVE DASHBOARD
}

\author{
Iwona ZDONEK \\ Silesian University of Technology; iwona.zdonek@polsl.pl, ORCID: 0000-0002-3377-0904
}

\begin{abstract}
Purpose: The objective of this paper is to make the reader aware of how to design dashboards dedicated to project indicator visualization so as to support their digestibility, usability (scannability) and customizability.
\end{abstract}

Design/methodology/approach: To achieve this goal, first, a literature review was conducted to identify principles for designing dashboards for this issue. Secondly, a real project management dashboard was built to investigate, in terms of technical issues, which interventions a project management dashboard designer should use to create a useful tool that would support the work of the project team.

Findings: The digestibility should address the following issues: 1) the optimal number of visualizations, 2) chart selections in a way that enables adaptation to data and assumed initiated action, 3) color design in the context of best contras, with color-blind users in mind. In addition, the usability (scannability) should be intuitive. Thus, elements related to time widget intervals and click to filter options have to be added. To provide the customizability, the dashboard should be designed to display project indicators tailored to the tasks of a particular project team. Originality/value: New in the paper is an examination of a dashboard digestibility, scannability, and customizability in the area of project indicator visualization.

Keywords: project management dashboard, business intelligence tools, exploiting Tableau in project management.

Category of the paper: a case study.

\section{Introduction}

Interactive visualizations such as business dashboards are an important approach that helps explaining and understanding large amounts of data, particularly in the light of data-driven decisions. Because of this, contemporary researchers and practitioners focus their attention on principles related to dashboard creation. Their articles describe different case studies related to developing dashboards for efficient data visualization at the healthcare facility level (Stadler et al., 2016), and at the level of data coming from prescriptions (Koa, and Chang, 2018). 
They explore how to develop dashboards that help users query, filter and analyze the tracking data gathered by the Major League Baseball (Lage et al., 2016), and how to use flow diagrams for understanding uncertainty (Vosough et al., 2019). They show how to visualize indicators in dashboards to enable comparisons among different cities (Santos et al., 2017), and how to visualize data for exploring the additive manufacturing solution space (Goguelin et al., 2017). We can also find articles related to general issues regarding rules of appropriate dashboard creation (Sedrakyan et al., 2019, Barr, 2014).

The objective of this paper is to allow the reader to understand how to design dashboards dedicated to project indicator visualization to support their digestibility, scannability, portability and customizability. To achieve this goal, first, a literature review was conducted to identify principles for designing dashboards. Secondly, a real project management dashboard was built to investigate, in terms of technical issues, which interventions a project management dashboard designer should use to create a useful tool supporting the work of a project team.

The paper is structured as follows. In section 1, the main methodological issues of the conducted research are presented. In section 2, the results of a literature review focused on project management dashboard creation are described. Section 3 presents the results of the mentioned principles as implemented. Finally, discussion and conclusion are drawn in section 4.

\section{Methodology}

Because the objective of this paper is to understand how to design dashboards dedicated to project indicator visualization, literature review and case study were conducted to identify the appropriate underlying principles. The literature review included articles from the Web of Science database, texts related to data visualization and blogs focused on dashboards design (including project management dashboard design). The papers coming from the Web of Science data covered a period of 5 years, from 2015 to 2019. The texts related to data visualization included the most important and the most often cited literature items as written by Edward Tuft, Stephen Few, Cole Nussbaumer Knaflic, and Natham Yau. They were published in the period from 2001 to 2015 . The articles coming from the blogs devoted to dashboard design covered the period from 2017 to 2019. These were published as papers referring to technical issues related to the software dedicated to business dashboard design.

The case study focused on implementing the principles, discovered in the literature review, into a real project management dashboard. In 2018 and 2019, the author of the article participated in a project financed by the European Union, the project main goal of which was the modernization of university-level education. The author's participation was related to taking part in the work of the project management team. 
One of the tasks that the project team had to do, was to create a project data visualization. Besides a financial context, an indicator context was requested by external and internal stakeholders. In this way, the demand arose for creating a project dashboard focused on the visualization of indicators. This dashboard was based on data generated by certain project tasks (11 of 31) that belonged to one of four project teams. The data covered a period of 14 months, from October 2018 to December 2019. It included the number of courses conducted in each department in the context of language, in semester and academic year categories. These were compared to the goals assigned to each department and the language category assumed at the beginning of the project.

The project management dashboard was created following the principles investigated during the literature review. The mentioned principles were implemented using the Tableau application. It let the author to consider, in terms of technical issues, which interventions a project management dashboard designer should use to create a useful tool for supporting the work of a project team.

\section{The literature review - looking for the project management dashboard creation principles}

According to S. Durcevic, "a dashboard in business is a tool used to manage all the business information from a single point of access. It helps managers and employees to keep track of the company's KPIs and utilizes business intelligence to help companies make data-driven decisions" (Durcevic, 2019a). Matheus and others add that "dashboards visualize a consolidated set data for a certain purpose which enables users to see what is happening and to initiate actions" (Matheus et al., 2018). Special dashboards created in Project Management are called project management dashboards. They can display metrics for project overall performance and progress. Their main object is highlighting particular problems that require further attention within the project team. These types of dashboards are created for individual departments that want to monitor the project progress and success.

Because project management dashboards are designed for individual departments, the metrics used on them depend primarily on the department and, of course, on project objectives. As with all dashboards, these are also created to inspire actions. For this reason, all metrics and KPIs used on them should encourage project team activity.

According to S. Durcevic (Durcevic, 2019b) and the Lucidchart team (Lucidchart, 2018), benefits arising from using project management dashboards are related, firstly, to better communication in a project team, secondly, to centralized data, and thirdly, to targeted information. However, the well-designed dashboard requires four important features. Firstly, it should be digestible - meaning that "data should be presented in an easy-to-digest 
format" (Lucidchart 2018) and "serve up insights in a way that lets value be extracted from them in seconds" (Durcevic 2019a). Secondly, the project management dashboard should be usable (scannable) - which means it has to be completely user-friendly (i.e. allow intuitive navigation and the conducting of data-driven tasks without technical fuss). Finally, it has to be customizable - which means displaying the most relevant KPIs and choosing designs or layouts that will prove the most helpful to the project team.

Providing all these features to the project management dashboard requires acquaintance of rules related to appropriate data visualization. In this regard, we can encounter a lot of texts that teach how to design visualizations (i.e., Tuft, 2001a, 2001b; Few, 2012; Yau, 2013, Knaflic, 2015). They focus on the rules for choosing the right chart to data, and for the avoidance of clutter in visualizations. They teach about the chart elements that focus user's attention and eliminate the exclusion of the color-blind users. The principles contained in these books are based on well-known Gestalt laws that are widely described on many blogs (i.e Meeks, 2017; Vidya, 2018; Craig, 2019; Kurcwald, 2019).

All mentioned project management dashboard features require the application of certain technical solutions. Among these are: click to filter options, drill-downs, time interval widgets, chart zooms, custom chart tooltips, hierarchical filters, dynamic text boxes or images and information tooltips (Durcevic 2019). Because dashboards display data in a visual form, it is worth mentioning that only some visualizations are recommended. Among them are simple text (i.e. KPIs), tables, heatmaps, scatterplots, line/slope graphs, vertical and horizontal bars, stacked vertical or horizontal bars, waterfalls and bullet graphs (Few, 2012; Knaflick, 2015; Oetting, 2019).

\section{The case study - implementation of principles}

\section{Customization}

The dashboard customization was the first dashboard feature that was implemented. The designed dashboard related to the project indicators connected to the number of conducted courses. Five persons from the project team were engaged in project processes appropriate to these indicators. Thus, the visualization of indicators concerning the number of courses was made based on the identification of basic metrics and their target values. The metrics were presented in the context of the most important dimensions such as project tasks, language categories and time intervals. The project tasks were related to particular departures. The language categories were concerned with courses conducted in English or Polish. The time intervals were applied to particular semesters or academic years. In this way, the dashboard included two visualizations presenting the level of the goal achievement in the context of project 
tasks. The first of these was presented by small colored icons showing the level of the goal achievement. The second showed the level of goal achievement using a bullet chart. Below both visualizations, bar charts were added that presented the course numbers in the context of the project task and language categories. These were accompanied by visualizations in the form of a table of course names as conducted in all departures (all project tasks). In the dashboard, the rule of click to filter was implemented. The majority of options related to this rule was placed on the right side of the dashboard. The dashboard user could also filter data by clicking on elements of the charts (Figure 1).

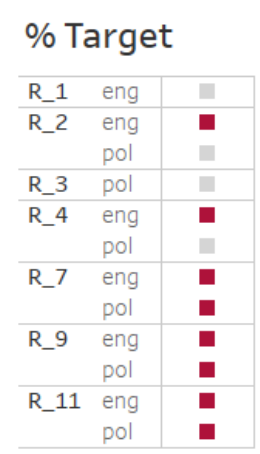

names

Advanced An.

Algorithms A.

Analiza I Proj.

Analiza Syst.

Analog-Mixe.

Applications.

Architectura.

Architectura.

Architecture .

Arithmetic 0 .

Automatyka.

Badania Ope.

Bezzałogow..

$<$ >

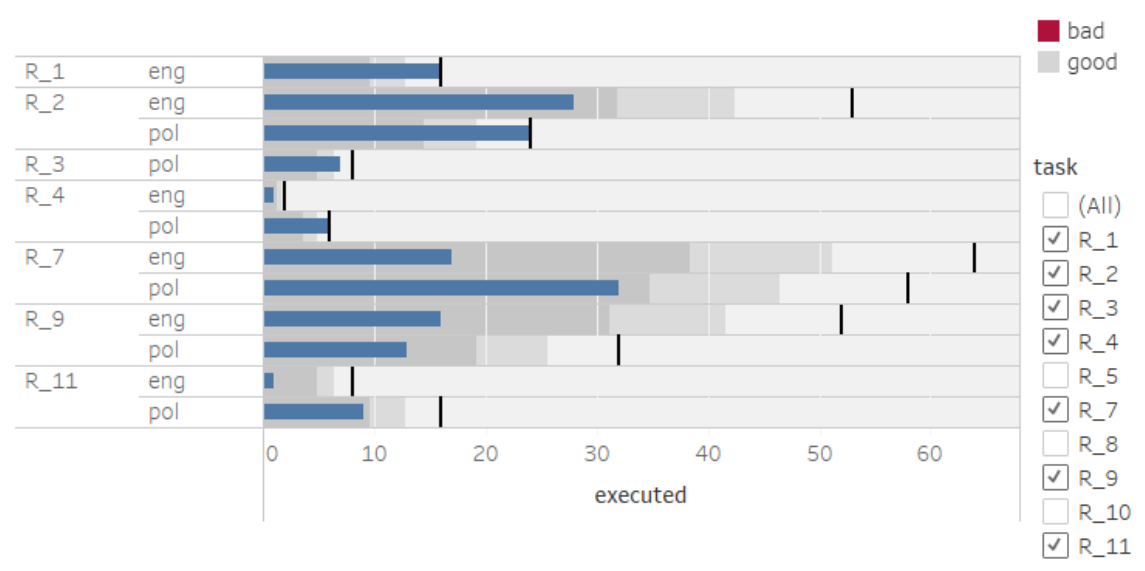

Courses in time intervals

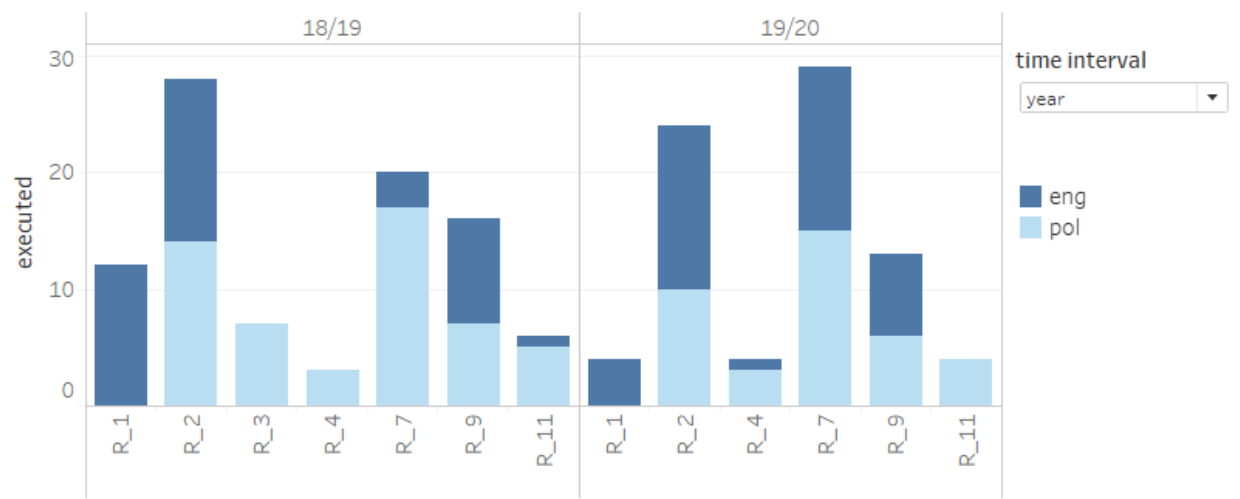

Figure 1. The visualization of indicators regarding the number of courses.

\section{Digestibility}

The implementation of the digestibility feature to the designed dashboard involved three main issues. Firstly, the dashboard could not be overloaded. For this reason, only four visualizations were designed. Secondly, the charts had to effectively visualize the data and indicate to the team project where action is necessary. Finally, the colors used in the visualizations had to be suitable for all users - including those who have problems with color perception. Since the designed dashboard should send signals and initiate actions, this function was implemented by two visualizations: a bullet chart preceded by a visualization presenting the goal achievement. This last utilized small colored icons. The choice of these visualizations 
was related to ensuring the digestibility of the designed dashboard. The bullet chart is a variation of a bar chart and is used to display the progress toward a curtained goal. Herein, the core data value is encoded by the length of the main bar in the middle of the chart. This presents the level of the project indicators achievement. The line marker that runs perpendicular to the orientation of the graph shows the goal and is used as a target marker to compare against the bar presenting the level of the project indicators achievement. The segmented colored bars behind the main bar are used to display qualitative range scores. Each color shade (three shades of grey) is used to assign a performance range rating. The visualization presenting the goal achievement by small colored icons is a modification of traffic light icon applications. Instead of red, orange and green colors, red, dark grey and light grey were used. This intervention was related to ensuring perception to color-blind users of signals sent by the dashboard (Figure 2).
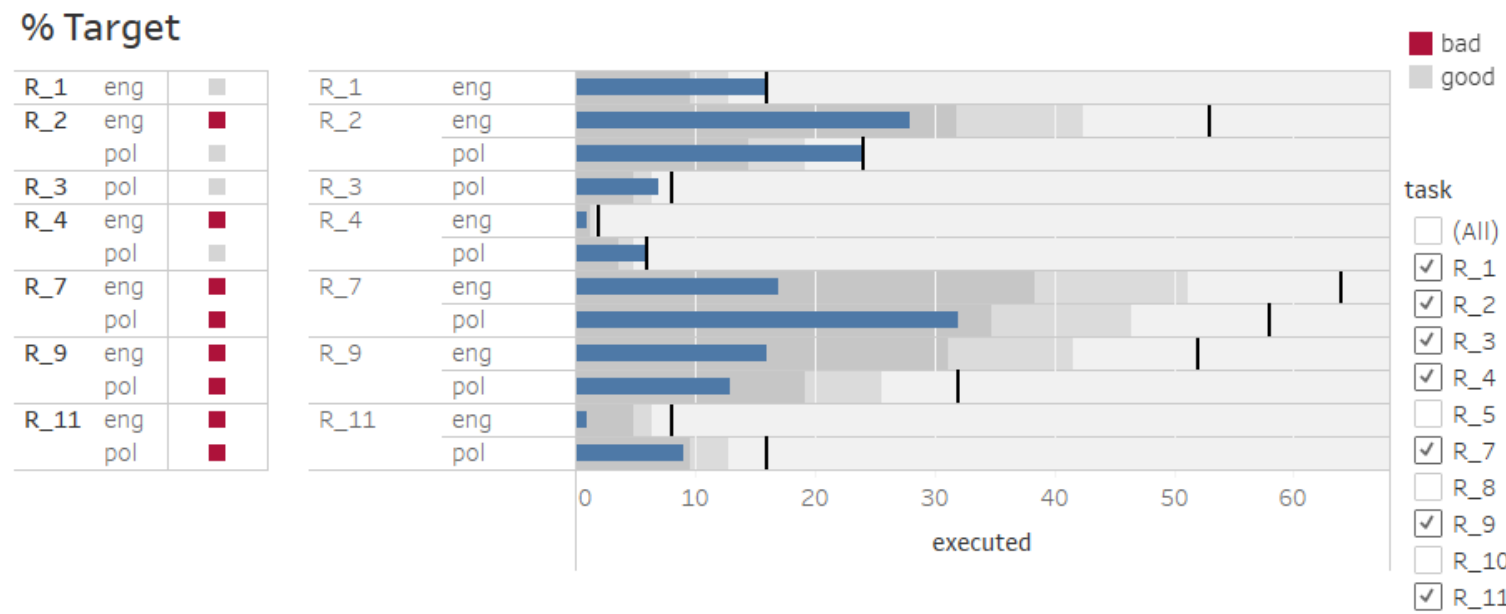

Figure 2. The implementation of the signal sending function.

The implementation of the functionality of the modified traffic light is related to the creation of a special measure that is based on the IF function. Afterwards, this measure is used as the element of visualization that determines the color. The variables that the IF function compares are depicted in the illustration below. The variable "executed" means the number of conducted courses, and the variable "target" denotes the planned target. The creation of this measure and using it as the element of the visualization determining the color is based on a similar mechanism that is known in MS Excel as conditional formatting.

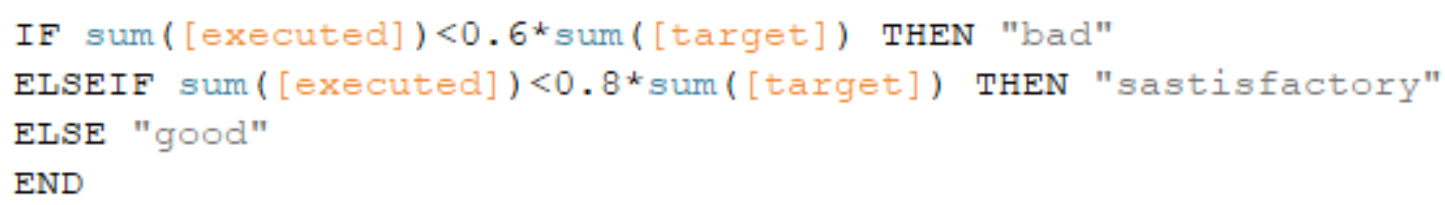

Figure 3. The implementation of the measure that enables the conditional formatting. 


\section{Usability}

This dashboard feature was implemented to include all elements related to the intuitive navigation. Firstly, the element that enables data presentation at different time intervals was designed. This element was put in place in the form of a drop-down list that allows the choice of the time interval. In this way, data can be displayed in semesters or years (Figure 4).

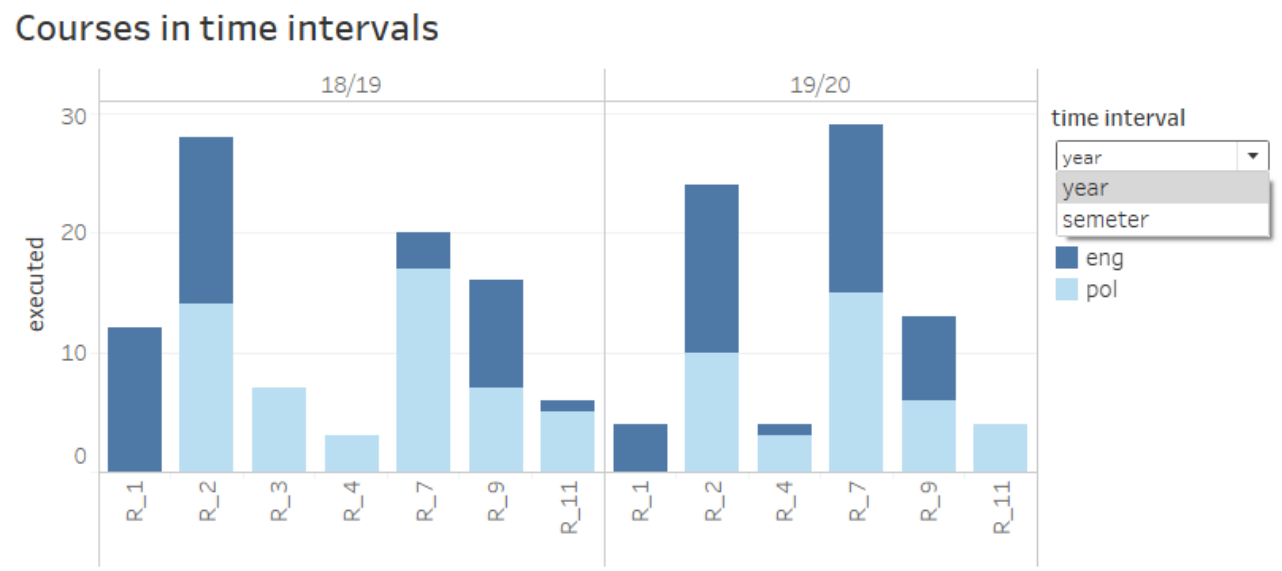

Figure 4. The interval time widget.

The implementation of this functionality requires, firstly, the special parameter creation, and secondly, using it to create a measure that enables the obtaining of a proper variable when choosing the parameter's option (Figure 5).

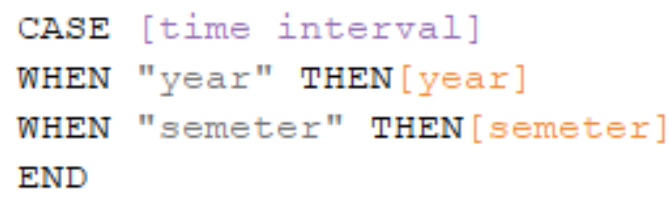

Figure 5. How to implement the option related to the time interval widget.

Secondly, a click to filter option was designed. This was executed on the basis of the bullet chart. The choice of a project task in it leads to filtering all visualizations in the dashboard. The bar chart then presents data only for the chosen task, and the table with course names depicts only courses related to that task (Figure 6). 

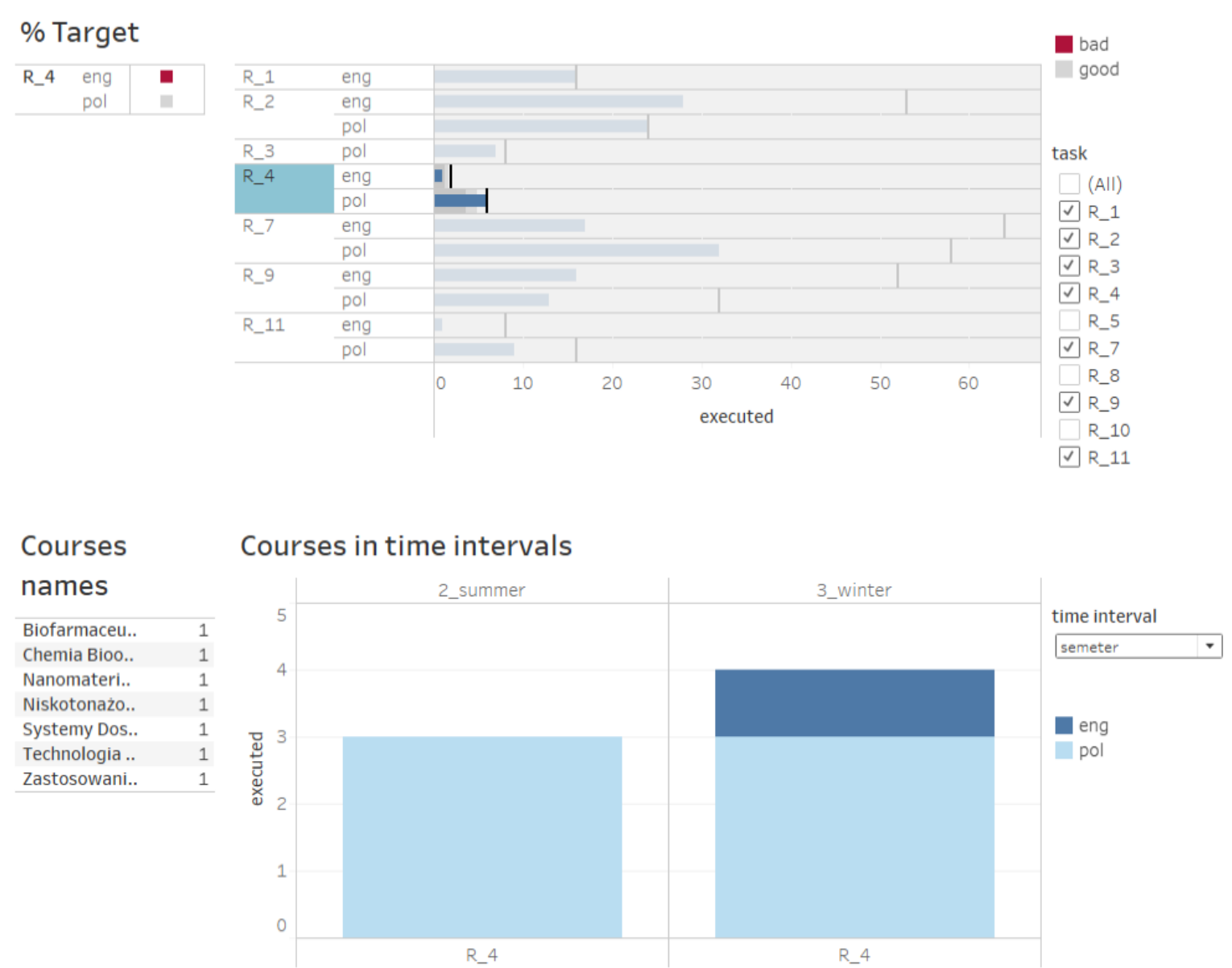

Figure 6. The click to filter option.

Finally, it is worth mentioning that all elements related to filtering were grouped on the right side of the designed dashboard.

\section{Discussion and conclusion}

The objective of this paper was to enable an understanding of how to design dashboards dedicated to project indicator visualization to support their digestibility, usability (scannability) and customizability. To achieve this goal, first, a literature review was conducted to identify principles for designing dashboards. Secondly, a real project management dashboard was built to investigate, in terms of technical issues, which interventions a project management dashboard designer should use to create a useful tool for supporting the work of the project team. After the analysis, the following conclusions can be made:

A. Customizability. The main purpose of the designed dashboard was to focus user's attention to the level of goal achievement in certain project tasks and to initiate actions that will help to maintain the appropriate level of indicators. Thus, the dashboard did not show all project tasks, but only those that were related to courses conducted in each 
department (called project tasks 1 to 11). In this way, the dashboard was dedicated to the project team that was responsible for the realization of these tasks and which implemented the customization of the designed dashboard.

B. Digestibility. This was enacted by generating the optimal number of visualizations (the dashboard contained 2 charts and 2 tables). Furthermore, the charts were chosen in a way that enabled adaptation to data and assumed initiated action. All colors used in the dashboard were designed in the context of best contrasting.

C. Usability (scannability). Usability was implemented through ensuring intuitive navigation. Thus, elements related to time widget intervals and click to filter options were added. Likewise, all filter options were grouped on the right side of the dashboard.

Related issues. To focus the user's attention, a traffic light visualization was designed. This choice followed the S. Barr rules (Barr, 2014) that recommended modification through using colors recommended for the inclusion of color-blind users. The function of signal sending realized by this chart was strengthened by visualization based on the bullet chart. The bullet chart choice was made through following principles that recommended this kind of chart to show, also in a quantitive way, the level of goal achievement. Herein, beyond the traffic light visualization being designed with color-blind users in mind (recommended by S Barr and C.N. Knaflic (Knaflic, 2015)), all best visualization notions were included in the designed dashboard. For this reason, all visualization were checked in terms of contrasting in shades of gray. Moreover, the color in all visualizations was used sparingly so as to not cause user cognitive burden. This approach followed the rule recommended by C.N. Knaflic. Although it does not happen very often, the principles (that a dashboard designer should respect) sometimes contradict each other and the designer must choose which of these rules should be followed. The mentioned contradiction was encountered during the bullet chart and the bar chart design. The Gestalt laws suggest sorting bars in a chart from largest to smallest, but following this rule could disturb the logical order in the data related to the project task that was ordered by numbers from 1 to 11 . For this reason, the bars were not sorted.

\section{References}

1. Barr, S. (2014). 3 Problems With KPI Traffic Light Dashboards. Retrieved from https://www.staceybarr.com/measure-up/3-problems-with-traditional-kpi-traffic-lights/.

2. Craig, W. (2019). How to Make Data Visualization Better with Gestalt Laws. Retrieved from https://www.webfx.com/blog/web-design/data-visualization-gestalt-laws/.

3. Durcevic, S. (2019a). Make Sure You Know The Difference Between Strategic, Analytical, Operational, And Tactical Dashboards. Retrieved from https://www.datapine.com/blog/ strategic-operational-analytical-tactical-dashboards/. 
4. Durcevic, S. (2019b). Your Guide To Project Management Dashboards - Examples \& Templates. Retrieved from https://www.datapine.com/blog/project-managementdashboards-examples-and-templates/.

5. Durcevic, S. (2019c). 10 Interactive Dashboard Features of Business Dashboards. Retrieved from https://www.datapine.com/blog/interactive-dashboard-features/.

6. Few, S.C. (2012). Show Me the Numbers. Analytics Press.

7. Goguelin, S., Flynn, J.M., Essink, W.P., Dhokia, V. (2017), A Data Visualization Dashboard for Exploring the Additive Manufacturing Solution Space. Procedia CIRP, 60, 93-198, doi: https://doi.org/10.1016/j.procir.2017.01.016.

8. Knaflic, C.N. (2015). Storytelling with data. New Jersey: Willey, Hoboken.

9. Koa, I., Chang, H. (2018). Interactive data visualization based on conventional statistical findings for antihypertensive prescriptions using National Health Insurance claims data. International Journal of Medical Informatics 116, 1-8, doi: https://doi.org/10.1016/ j.ijmedinf.2018.05.003.

10. Kurcwald, K. (2019). 5 Rules for Clarity in Data Visualization. Retrieved from https://www.monterail.com/blog/rules-of-data-visualization.

11. Lage, M., Ono, J.P., Cervone, D., Chiang, J., Dietrich, C., Sila, C.T. (2016). StatCast dashboard: exploration of Spatiotemporal baseball data. IEEE Computer Graphics and Applications, 36, 5. Retrieved from https://ieeexplore.ieee.org/abstract/document/7579419/ metrics\#metrics.

12. Lucidchart (2018). How to Use a Project Dashboard to Keep Your Team on Track. Retrieved from https://www.lucidchart.com/blog/project-management-dashboards.

13. Matheus, R., Janssen, M., Maheshwari, D. (2018). Data science empowering the public: Data-driven dashboards for transparent and accountable decision-making in smart cities. Government Information Quarterly. Retrieved from https://www.sciencedirect.com/ science/article/pii/S0740624X18300303.

14. Meeks, E. (2017). Gestalt Principles for Data Visualization. Retrieved from https://medium.com/@Elijah_Meeks/gestalt-principles-for-data-visualization59f18f20bd40.

15. Oetting, J. (2019). Data Visualization 101: How to Choose the Right Chart or Graph for Your Data. Retrieved from https://blog.hubspot.com/marketing/types-of-graphs-for-datavisualization.

16. Santos, H., Dantas, V., Furtado, V., Pinheiro, P., McGuinness, D.L. (2017). From Data to City Indicators: A Knowledge Graph for Supporting Automatic Generation of Dashboards. In: E. Blomqvist, D. Maynard, A. Gangemi, R. Hoekstra, P. Hitzler, O. Hartig (eds.), The Semantic Web. ESWC 2017. Lecture Notes in Computer Science, 10250. Cham: Springer. doi: https://doi.org/10.1007/978-3-319-58451-5_7. 
17. Sedrakyan, G., Mannens, E., Verbert, K. (2019). Guiding the choice of learning dashboard visualizations: Linking dashboard design and data visualization concepts. Journal of Visual Languages and Computing 50, 19-38, doi: https://doi.org/10.1016/j.jvlc.2018.11.002.

18. Stadler, J.G., Donlon, K., Siewert, J.D., Franken, T., Lewis, N.E. (2016). Improving the Efficiency and Ease of Healthcare Analysis Through Use of Data Visualization Dashboards. Big Data, 4, 2, doi: https://doi.org/10.1089/big.2015.0059.

19. Tuft, E.R.(2001b). Envisioning Information. Cheshire, CT: Graphics Press.

20. Tufte, E.R. (2001a). The Visual Display of Quantitative Information. Cheshire, CT: Graphics Press.

21. Vidya (2018). Gestalt Laws Applied to Data Visualization. Retrieved from http://daydreamingnumbers.com/concepts/gestalt-laws-data-visualization/.

22. Vosough, Z., Kammer, D., Keck, M., Groh, R. (2019). Visualization approaches for understanding uncertainty in flow diagrams. Journal of Visual Languages and Computing, 52, 44-54, doi: https://doi.org/10.1016/j.cola.2019.03.002.

23. Yau, N. (2013). Data points. Willey. 